ROTATION SURFACES OF CONSTANT CURVATURe. [July,

set of postulates for Boolean algebras where the remaining postulates of the set are already free from all implicational relationships among themselves.

Massachusetts Institute of Technology.

\title{
ROTATION SURFACES OF CONSTANT CURVATURE IN SPACE OF FOUR DIMENSIONS.
}

\author{
BY PROFESSOR C. L. E. MOORE.
}

(Read before the American Mathematical Society April 24, 1920.)

1. IN space of four dimensions there are two special rotations each of which has circles for path curves. In this note I shall discuss the surfaces generated by these special rotations which have constant curvature. The first type is given by the equations

$$
\begin{gathered}
X=x \cos t-y \sin t, \quad Y=x \sin t+y \cos t, \\
Z=z, \quad W=w .
\end{gathered}
$$

This rotation leaves each point of the $z w$-plane invariant and any plane completely perpendicular to it is left invariant as a plane but not point for point. The rotation then is simply isomorphic with a rotation in the $x y$-plane.*

If the curve

$$
x=x(s), \quad y=y(s), \quad z=z(s), \quad w=w(s),
$$

where $s$ denotes arc length measured from a fixed point, is rotated, equations (1) are the parametric equations of the surface generated. The parameter curves $s=$ const., $t=$ const. will be orthogonal if

$$
x y^{\prime}-x^{\prime} y=0 \text { or } y=k x,
$$

where primes denote derivatives with respect to $s$. Hence the meridian curves (orthogonal trajectories of the path curves) on a surface generated by rotation (1), lie in a 3-space which contains the absolutely invariant plane. If the meridian curve

\footnotetext{
* Phillips and Moore, "Rotations in space of even dimensions," Proceedings Amer. Academy, vol. 55.
} 
is a plane curve its plane must lie in one of these 3-spaces and consequently cut the $z w$-plane in a line. It will then also cut a set of invariant planes which are completely perpendicular to the $z w$-plane in lines. A plane which cuts the $z w$-plane in a line will then generate a 3 -space by the rotation (1). Hence the surfaces of rotation, generated by (1), for which the meridians are plane curves must lie in a 3-space. Such surfaces of constant curvature are already well known.

If the meridians are not plane curves they must satisfy (2), and for simplicity we will assume that it lies in the $x z w$ space. The element of arc on such a surface is

$$
d \sigma^{2}=d s^{2}+x^{2} d t^{2}
$$

where $s$ denotes the arc on the meridian curve and $\sigma$ the arc of any curve traced on the surface of rotation. If the surface has constant positive curvature,*

The integral of this is

$$
\frac{1}{a^{2}}=-\frac{1}{x} \frac{d^{2} x}{d t^{2}}
$$

$$
x=c \cos (s / a+b) .
$$

By properly choosing the reference system $b$ can be made to vanish. This we will assume done. We can then write

$$
\begin{aligned}
& d x^{2}+d z^{2}+d w^{2}=d s^{2} \quad \text { or } \\
& d z^{2}+d w^{2}=\left(1-\frac{c^{2}}{a^{2}} \sin ^{2} \frac{s}{a}\right) d s^{2} .
\end{aligned}
$$

For a rotation surface in 3 -space equation (5) reduces to

$$
d z^{2}=\left(1-\frac{c^{2}}{a^{2}} \sin ^{2} \frac{s}{a}\right) d s^{2},
$$

the solution of which is well known. For the case here considered, $d z^{2}+d w^{2}=d s^{2}$ is the square of the element of arc of the projection of the meridian curve on the $z w$-plane. Equation (6) then becomes

$$
d s_{1}{ }^{2}=\left(1-\frac{c^{2}}{a^{2}} \sin ^{2} \frac{s}{a}\right) d s^{2} .
$$

* Eisenhart, Differential Geometry, p. 270. 
The solution for $s_{1}$ in terms of $s$ is the same as the solution of (6) for $z$ in terms of $s$. The projection $s_{1}$ then bears the same relation to $x$ that $z$ does for a rotation surface of constant positive curvature in 3-space. Hence to obtain curves in the $x z w$-space which can be used as meridian curves of a surface of revolution of constant positive curvature, trace on the zw-plane curves $(c)$ which will generate such surfaces. Then roll this plane into a cylinder with elements parallel to $O X$, making $O Z$ roll into the section of the cylinder in the zw-plane. The curves into which the curves (c) roll are the curves sought.

2 . The second kind of rotation which has circular path curves is

$$
\begin{aligned}
& X=x \cos t-y \sin t, \quad Y=x \sin t+y \cos t, \\
& Z=z \cos t-w \sin t, \quad W=z \sin t+w \cos t .
\end{aligned}
$$

This rotation leaves $\infty^{2}$ planes of a linear congruence invariant.* These can be arranged into pairs of completely perpendicular planes. If the curve $(c)$ is rotated (7) will be the parametric equations of the resulting surface. The parameter curves on this surface will be orthogonal if

$$
x^{\prime} y-x y^{\prime}+z^{\prime} w-z w^{\prime}=0 .
$$

Now by this rotation $\dagger$ if a line cuts one path curve orthogonally it will cut all orthogonally and consequently must lie in a plane which cuts two completely perpendicular invariant planes in lines. Therefore the solutions of (8) must lie on cones whose tangent planes cut a pair of completely perpendicular invariant planes in lines. This equation shows that no 3-space curve lying in a 3 -space passing through the origin can be used as a meridian curve. For by choosing the axes properly such a 3 -space can be represented by $x=0$ and consequently, from (8), $z=k w$ and hence the curve must be plane. If the curve is plane it must lie in a plane cutting two completely perpendicular invariant planes in lines. We will discuss this case first.

Let the curve lie in the $x z$-plane. Equations (7) then become

$$
X=x \cos t, \quad Y=x \sin t, \quad Z=z \cos t, \quad W=z \sin t .
$$

* Moore, "Rotations in hyperspace," Proceedings Amer. Academy, vol. 53.

† Moore, "Surfaces of rotation in space of four dimensions," Annals of Math., vol. 21. 
1920.] ROTATION SURFACES OF CONSTANT CURVATURE. 457

The element of arc of the surface formed by rotating $x=x(s)$, $z=z(s)$ is

$$
d \sigma^{2}=d s^{2}+\left(x^{2}+z^{2}\right) d t^{2} .
$$

If the surface has constant positive curvature, then

$$
\rho=\sqrt{x^{2}+z^{2}}=c \cos \frac{s}{a} .
$$

Using polar coordinates in the $x z$-plane, we then have

$$
d \rho^{2}+\rho^{2} d \theta^{2}=\frac{a^{2} d \rho^{2}}{c^{2}-\rho^{2}} \text { or } \frac{1}{\rho} \sqrt{\frac{\rho^{2}+a^{2}-c^{2}}{c^{2}-\rho^{2}}} d \rho=d \theta .
$$

Just as in three dimensions, there are three types of curves depending on the relations between $a$ and $c$.

(a) If $a=c$, the solution is

$$
\rho=a \sin (\theta+b) .
$$

Thus any circle of radius a/ 2 passing through the origin will rotate, by (7), into a surface of constant positive curvature a. If the circle has its center at the origin, it will generate a developable surface as is at once evident from (9).

(b) If $a<c$, the solution is

$$
\begin{aligned}
\frac{1}{2} \sin ^{-1} & \frac{2 \rho^{2}+a^{2}-2 c^{2}}{a^{2}} \\
& -\frac{1}{2} \sqrt{\frac{c^{2}-a^{2}}{c^{2}}} \sin ^{-1} \frac{2 c^{2}\left(a^{2}-c^{2}\right)+\left(2 c^{2}-a^{2}\right) \rho^{2}}{c^{2} \rho^{2}}=\theta+b .
\end{aligned}
$$

(c) If $a>c$, the solution is

$$
\begin{aligned}
& \frac{1}{2} \sin ^{-1} \frac{2 \rho^{2}+a^{2}-2 c^{2}}{a^{2}} \\
& \quad-\frac{1}{2} \sqrt{\frac{a^{2}-c^{2}}{c^{2}}} \cosh ^{-1} \frac{2 c^{2}\left(a^{2}-c^{2}\right)+\left(2 c^{2}-a^{2}\right) \rho^{2}}{c^{2} \rho^{2}}=\theta+b .
\end{aligned}
$$

In cases $(a)$ and $(b)$ the solutions can be written in finite form, while the corresponding solutions for 3-space can be written in parametric form only by the use of elliptic functions.*

* See Bianchi, vol. 1, p. 233. 
To determine the form of these curves we return to equation (11) and write it in the form

$$
\sqrt{\frac{\rho^{2}+a^{2}-c^{2}}{c^{2}-\rho^{2}}}=\frac{\rho d \theta}{d \rho}=\tan \psi,
$$

where $\psi$ is the angle between the tangent line and the radius vector.

If $a<c$, this last equation shows that $c^{2}-a^{2}<\rho^{2}<c^{2}$ and hence the curve lies between two concentric circles of radii $\sqrt{c^{2}-a^{2}}$ and $c$. Also at the inner circle $\psi$ is zero and at the outer circle $\pi / 2$. This curve then consists of segments which start perpendicular to the inner circle, touch the outer circle and return perpendicular to the inner circle. If $a>c$ the curve is in the form of a loop which crosses at the origin and is tangent to the circle $\rho=c$. For given values of $a$ and $c$ equation (11) shows that all curves cut the circle $\rho=$ const. at the same angle and have the same radius of curvature at the points of intersection. The curves then are symmetrical about the line joining the origin to the point of tangency with $\rho=c$. All curves are obtained by rotating a given one about the origin.

3. If the surface is one of constant negative curvature,

$$
\frac{1}{a^{2}}=\frac{1}{\rho} \frac{d^{2} \rho}{d t^{2}} .
$$

Hence $\rho=c_{1} \cosh s / a+c_{2} \sinh s / a$.

The three cases to consider are $c_{1}=0 ; c_{2}=0 ; c_{1}=c_{2}$. The square of the element of arc in each of these three cases is

$$
\begin{aligned}
& d \sigma^{2}=d s^{2}+c^{2} \cosh ^{2} s / a d t^{2}, \\
& d \sigma^{2}=d s^{2}+c^{2} \sinh ^{2} s / a d t^{2}, \\
& d \sigma^{2}=d s^{2}+c^{2} e^{2 s / a} d t^{2} .
\end{aligned}
$$

Any case other than these may be obtained by taking for $\rho$ either of the values $\cosh (s / a+b), \sinh (s / a+b)$ where $b$ is constant. For the first case, following the previous discussion, we have

$$
d \rho^{2}+\rho^{2} d \theta^{2}=\frac{a^{2} d \rho^{2}}{\rho^{2}-c^{2}} \text { or } \sqrt{\frac{\rho^{2}-c^{2}-a^{2}}{c^{2}-\rho^{2}}}=\frac{\rho d \theta}{d \rho} .
$$

In this case also the equation is readily integrated in terms 
of elementary functions, but we will obtain the properties of the curve from equation (12) itself. The first thing observed is that $c^{2}<\rho^{2}<c^{2}+a^{2}$. Hence the curve does not pass through the origin for any value of $c$ but lies between two concentric circles with center at the origin as before. In this case however the curve is tangent to the inner circle and perpendicular to the outer circle. This corresponds to the pseudospherical surface of hyperbolic type.

In case (ii) the differential equation is

$$
\sqrt{\frac{a^{2}-c^{2}-\rho^{2}}{c^{2}+\rho^{2}}}=\frac{\rho d \theta}{d \rho} .
$$

In this case, then, $a>c$ and $\rho^{2}<a^{2}-c^{2}$. The curve then lies inside the circle $\rho=\sqrt{a^{2}-c^{2}}$ to which it is perpendicular. As the value of $\rho$ decreases, the value of $\psi$ approaches $\tan ^{-1} \sqrt{\left(a^{2}-c^{2}\right) / a^{2}}$ and therefore the curve approaches the origin, cutting all the radius vectors at the above angle.

In case (iii) we have

$$
\frac{1}{\rho} \sqrt{a^{2}-\rho^{2}}=\frac{\rho d \theta}{d \rho} .
$$

The curve then lies inside the circle $\rho=a$ which it cuts perpendicularly. As $\rho$ decreases, $\psi$ approaches $\pi / 2$. This curve then approaches the origin, cutting the radius vectors at right angles. This is the limiting case of either of the above. If any of the above curves are rotated about the origin in the $x z$-plane, when rotated by (7) they will still generate a surface of constant negative curvature. The forms of all these differentials lend themselves readily to graphical solution so that a picture of the curves is easily obtained.

4. In case of the general rotation (7) the square of the element of arc is

$$
d \sigma^{2}=d s^{2}+\left(x^{2}+y^{2}+z^{2}+w^{2}\right) d t^{2}
$$

and if the surface is one of constant positive curvature,

$$
\rho=\sqrt{x^{2}+y^{2}+z^{2}+w^{2}}=c \cos \frac{s}{a} .
$$

Consequently the distance $\rho$ of the points from the origin is a function of $s$. Then if we have a curve (c) satisfying 
equation (14) and unroll the cone which projects this curve from the origin, on the $x z$-plane, the curve into which $(c)$ goes will satisfy (10) and hence will rotate into a surface of constant positive curvature. Hence the curves of 4-space which rotate by (7) into surfaces of constant positive curvature are obtained by tracing on the xz-plane curves (c) which rotate by (7) into surfaces of constant positive curvature, and then rolling the xz-plane into a cone with vertex at the origin. The curves into which the curves (c) go are those sought. Of course to obtain curves which cut the path curves orthogonally the above cones must be such that each of their tangent planes cuts a pair of completely perpendicular planes in lines. Curves which generate surfaces of constant negative curvature are obtained in a similar manner.

Massachusetts Institute of Technology.

\section{SHORTER NOTICES.}

The Mystery of Space. A study of the hyperspace movement in the light of the evolution of new psychic faculties, and an inquiry into the genesis and essential nature of space. By Robert T. Browne. New York, E. P. Dutton and Company, 1919. 8 vo. 395 pp. $\$ 4.00$.

THIs book is a mixture of cosmogony, psychology, and geometry; heralded in a recent flyer as "an epoch-making work."

With the cosmogony and psychology we can have little to do. Anyone obviously has a perfect right to philosophize about the universe and the true nature of space as much as he pleases and to dress his philosophy in Greek nomenclature to give it a scientific aspect if he chooses. But the reader may be pardoned if he is tempted to compare the periodic wanderings of Mr. Browne's "monopyknon," from chaos through seven stages of "pyknosis" before emerging into physical being, and thence through sentient, mental, and spiritual stages back to chaos again, with Goethe's story of the Homunculus; and to assert that the one is as mediaeval in character as the other was intended to be. Nor can one deny to the author the right to hold and to defend, if he can, the theory that humanity will one day develop to the point where, 